\title{
Tumour-Associated Hypoxia: Can We Give Chimeric Antigen Receptor T Cells More Breathing Space?
}

CAR-T cells are already a targeted immunotherapy. The developmen of CAR-engineered T cells that are exclusively expressed or activated under conditions of profound hypoxia, a feature of solid tumours, would increase the efficiency of this approach. In dual-targeted EDITOR'S

CAR-T, the therapy would utilise the antigens from one side and the hypoxia from the other side.

\section{Prof Ahmad Awada}

Jules Bordet Institute, Brussels, Belgium

\section{Authors:}

Disclosure:

Acknowledgements:

Received:

Accepted:

Keywords:

Citation:
*Karen I Larios Martinez, ${ }^{1}$ Paris Kosti,, Anna Schurich, ${ }^{2}$ James N Arnold, ${ }^{1}$ John Maher, 1,3

1. School of Cancer and Pharmaceutical Sciences, King's College London, Faculty of Life Sciences and Medicine, Guy's Campus, London, UK

2. School of Immunology and Microbial Sciences, King's College London, Faculty of Life Sciences and Medicine, Guy's Campus, London, UK

3. Department of Immunology, Eastbourne Hospital, East Sussex, UK

4. Department of Clinical Immunology and Allergy, King's College Hospital National Health Service (NHS) Foundation Trust, London, UK

5. Leucid Bio, Guy's Hospital, London, UK

*Correspondence to karen.i.larios_martinez@kcl.ac.uk

Dr Larios Martinez reports financial support from Consejo Nacional de Ciencia y Tecnologia (CONACYT) and Leucid Bio. Dr Maher is founder, chief scientific officer, and shareholder in Leucid Bio; he reports personal consultancy fees from Leucid Bio during the conduct of the study and grants from Leucid Bio, outside the submitted work; in addition, Dr Maher is co-inventor on a patent entitled "Hypoxia regulated CARs" pending to King's College London. The other authors have declared no conflicts of interest.

All authors contributed equally to this manuscript.

25.03 .20

14.04 .20

Adoptive cell therapy, chimeric antigen receptor (CAR), CAR-T cells, hypoxiainducible factor-1- $\alpha$ (HIF1- $\alpha$ ), hypoxia-responsive elements (HRE), hypoxia, on-target off-tumour, immunotherapy, oncology, oxygen-sensitive CAR.

EMJ. 2020;5[3]:30-37.

\section{Abstract}

Immunotherapy using chimeric antigen receptor (CAR)-engineered $\mathrm{T}$ cells has encountered important limitations in the transition of their use from liquid to solid tumours. Success is dependent upon T-cell trafficking to, and functional persistence within, tumours that often present a metabolically 
and immunologically hostile microenvironment. Moreover, CAR targets that are tumour specific are extremely scarce. To address these issues, several strategies have been proposed to improve both tumour selectivity and safety. One approach involves the engineering of CAR-T cells that only deploy their effector function at tumour sites. Conceptually, a solution for this exploits the oxygen-limited nature of advanced tumour deposits through the engineering of CAR that are exclusively expressed or activated under conditions of profound hypoxia. T cells have a complex inter-relationship with oxygen, which also needs to be factored into the refinement of these technologies. Ideally, oxygen-sensing CAR should only function when oxygen tension is below $2 \%$, as is commonly the case in solid tumours but rare in healthy tissue. Successful advancement of such technologies presents opportunities for solid tumour immunotherapy because it should broaden the target repertoire that may safely be exploited in this context.

\section{INTRODUCTION: THE CHALLENGE OF SOLID TUMOURS}

Chimeric antigen receptors (CAR) are fusion molecules that couple the binding of a native tumour-associated target to the delivery of a bespoke T-cell activating signal. To extend the success of CAR-T cells from the haematological to the solid tumour setting, a number of key challenges have hindered progress.' Firstly, there are numerous physical constraints that hinder CAR-T cell recruitment, activation, proliferation, and persistence within solid tumour deposits. Secondly, CAR-T cells that do manage to navigate into the solid tumour microenvironment must then operate in the face of the adverse metabolic conditions at the site, notably hypoxia, low $\mathrm{pH}$, elevated lactate concentration, and deprivation of nutrients such as glucose and amino acids. ${ }^{2}$ Even in the setting of more amenable haematological malignancies such as B-cell leukaemias and lymphomas, important safety concerns include potentially lethal cytokine release syndrome and neurotoxicity. In solid tumours, the relative absence of truly tumourselective targets means that on-target, off-tumour toxicity is a further major concern that has caused morbidity and death of some patients. ${ }^{3}$

Numerous strategies have been developed to improve the therapeutic index of CAR-T cell immunotherapy. 4,5 These include the intrinsic modification of CAR architecture, engineering of systems to control CAR-T cell persistence, and use of loco-regional delivery systems to minimise systemic exposure to these cells. ${ }^{6,7}$ Exemplifying this, a recent clinical trial has demonstrated the safety and efficacy of mRNA-transfected c-Met-CAR-T cells following their intratumoural administration, thereby achieving transient expression of a potentially toxic CAR primarily at the site of disease. ${ }^{8}$ Similarly, intratumoural delivery of panErbB CAR-T cells has been safely employed in the treatment of patients with relapsed or refractory and locally advanced head and neck cancers. 9 More complex strategies that set out to ensure safety include the use of suicide genes, ${ }^{10}$ inhibitory CAR," dual antigentargeted systems, ${ }^{12}$ combinatorial SynNotch CAR, ${ }^{13}$ and switchable CAR. ${ }^{14-17}$ To maximise success and safety requires a system that tightly restricts the activation of CAR-T cells to the solid tumour microenvironment.

In 2019, the Nobel Prize in Physiology or Medicine was awarded jointly to William Kaelin, Peter Ratcliffe, and Gregg Semenza for their work which characterised how cells sense and adapt to ambient oxygen levels. Given the profoundly hypoxic nature of many solid tumours, this paper considers the potential effects of hypoxia on CAR-T cells and how these may be harnessed to enhance the tumour specificity of these cells.

\section{HYPOXIA OF THE TUMOUR MICROENVIRONMENT}

Hypoxia refers to a low concentration of oxygen and is a frequent attribute of solid tumours. ${ }^{18}$ It commonly results from rapid tumour growth that outstrips an inadequate and often abnormal blood supply. Hypoxia has been associated with tumour cell invasiveness, metastasis, epithelial to mesenchymal transition, maintenance of cancer stem cells, and resistance to both radiation and cytotoxic chemotherapy. ${ }^{19-21}$ While oxygen tension in normal tissues is generally $\geq 5 \%$ and reaches approximately $14 \%$ in the lung, levels fall to below $2 \%$ in solid tumours and in 
chronically inflamed tissues. ${ }^{22}$ Oxygen plays a fundamental role in many metabolic processes and cells must adapt nimbly to fluctuating levels of this nutrient in order to remain viable. ${ }^{23-25}$ The key regulators of oxygen homeostasis are hypoxia-inducible factors (HIF): HIF-1, HIF2 , and HIF-3. ${ }^{26}$ While the importance of HIF3 is less apparent, HIF-1 and HIF-2 operate as transcriptional regulators of an overlapping set of genes that mediate the hypoxic adaptive response. ${ }^{27}$

HIF are heterodimers comprising a stably expressed $\beta$ subunit and a hypoxia-dependent $\alpha$ subunit. At normal oxygen levels, HIF-la is rapidly degraded. This process is dependent upon the hydroxylation of proline residues found in the oxygen-dependent degradation domain (ODD) of HIF-1a by the enzyme, prolyl hydroxylase. Upon hydroxylation, the von Hippel-Lindau protein (VHL) binds and orchestrates HIFla ubiquitination followed by proteasomal degradation. ${ }^{26}$ Conversely, under hypoxic conditions, the HIF-1a protein accumulates, translocates to the nucleus, and dimerises with the HIF-1ß subunit, forming the HIF-1 complex. The latter can then bind to hypoxia-responsive elements in the promoter region and increase transcription of specific hypoxia-induced genes. ${ }^{28}$

It has been argued that hypoxia represents the best validated cancer-associated feature that has yet to be exploited clinically. ${ }^{29}$ A wide number of experimental treatments are focussed on harnessing tumour-associated hypoxia, including prodrugs ${ }^{30}$ and HIF inhibitors. ${ }^{31}$ CAR-T cell immunotherapy strategists also need to consider the challenge and potential opportunity presented by the profoundly hypoxic nature of many solid tumours. Recent studies have investigated the effects of oxygen deprivation on CAR-T cells and some research groups have gone further to exploit hypoxia by developing oxygen-sensing CAR-T cells.

\section{CAR-T CELLS AND HYPOXIA}

Little is known about how CAR-T cells behave in an hypoxic environment. However, hypoxia is known to exert several effects on unmodified $\mathrm{T}$ cells, including the induction of apoptosis, compromised activation, and upregulated expression of inhibitory receptors. 18,32 Nonetheless, there is evidence that these inhibitory effects of hypoxia are not generalisable for all T-cell subsets. It has been argued that hypoxia favours the differentiation of Th9, Th17, and Th22 cells while hindering the differentiation of Th1 and cluster of differentiation-8 (CD8) effector $T$ cells, while data on the effect on regulatory $T$ cells are conflicting. ${ }^{18,33}$ Moreover, Xu et al. ${ }^{34}$ showed that hypoxia enhanced the proliferation, survival, and cytolytic activity of effector memory $T$ cells, in contrast to its inhibitory effects on naïve and central memory $T$ cells. This effect was attributed to elevated glycolytic activity, accompanied by enhanced HIF-la expression. Hypoxia enhanced the cytotoxic activity of a CAR directed against the GD2 ganglioside, associated with increased degranulation of the CAR-T cells upon target recognition. ${ }^{34}$ Furthermore, antigen-specific CAR-T cells that were mobilised into hypoxic tumour areas were more proliferative than CAR-T cells in normoxic areas, when evaluated in two in vivo models. ${ }^{34,35}$

Berahovich et al. ${ }^{36}$ also explored the effects of hypoxia on CAR-T cells targeted against B-cell maturation antigen (BCMA) and CD19. They observed that hypoxia diminished the expansion of both CAR- and non-transduced T cells. ${ }^{36}$ Differentiation of both BCMA and CD19 CAR-T cells was also affected, switching from central memory towards an effector memory phenotype. They also observed an increase in the CD4:CD8 CAR-T cell ratio, suggesting that CD8+ CAR-T cell expansion is more dependent on oxygen. ${ }^{36}$ While cytolytic activity of CAR-T cells that were expanded in 5\% oxygen was increased, these cells manifested a significant reduction in the production of interferon- $y$, IL-2, IL-6, and granzyme B, with programmed cell death protein 1 levels unaffected compared to CAR-T cells expanded in atmospheric oxygen levels. ${ }^{36}$ Consistency of findings between CD19 and BCMA-targeted receptors suggests that the effects of hypoxia were CAR-independent.

These in vitro data provide an insight into the way CAR-T cells function in a microenvironment restricted of oxygen, simulating that found in the tumour microenvironment. These findings also provide the basis for design of advanced CAR systems that actively exploit tumour-associated hypoxia for therapeutic gain. 


\section{RESTRICTING CAR-T CELL FUNCTION TO HYPOXIC ENVIRONMENTS}

The first attempt to exploit the hypoxic tumour microenvironment to regulate CAR expression was reported by Ang et al. ${ }^{37}$ In an abstract publication, they described the use of the 'Sleeping Beauty' transposon system to express a second generation (CD28+CD3ろ) anti-CD19 CAR fused to an ODD to restrict CAR expression to conditions of hypoxia. No expression of the ODD-containing CAR construct was seen at atmospheric oxygen (referred to as normoxia hereafter), while cell-surface CAR expression was detectable following the transfer of cells to $1 \%$ oxygen. ${ }^{37}$

The following year, this group described an oxygen-sensitive c-Met-specific CAR that utilised a fused ODD domain derived from HIF-la (Figure $1 \mathrm{~A}-\mathrm{B}){ }^{38}$ Like its predecessor, this CAR was expressed using the Sleeping Beauty transposon system. Cell-surface expression of the CAR and cytolytic activity against c-Met-engineered target cells were both strictly dependent upon hypoxic conditions. ${ }^{39}$ In a related approach, the clinical-stage biopharmaceutical company, Cellectis, Paris, France, described a method to create a multi-input signal-sensitive $T$ cell. The method involved the application of a logic AND gate whereby two independent stimuli as input signals are required for CAR activation to occur. To exploit hypoxia as one such input signal, the CAR was engineered to contain an ODD. By this means, cognate ligand engagement was restricted to conditions of hypoxia. ${ }^{40}$

The hypoxia-exploiting CAR described in this paper had a multichain (mc)CAR architecture (Figure 2). The latter was based on the highaffinity IgE receptor (FcERI), ${ }^{41}$ a configuration that had earlier been designed to regulate CAR activity in a small molecule-dependent manner. ${ }^{14}$ The mcCAR was engineered by replacing the extracellular domain of the a chain for a singlechain variable fragment fused to a hinge domain from the CD8 $\alpha$ chain. The native domain of the $\beta$ chain was substituted with a 4-1BB domain while the $\mathrm{CD} 3 \zeta$ chain was appended to the $\mathrm{Y}$ chain endodomain. Three CAR were designed as follows. HIF-CAR1 was engineered by fusing the ODD sequence (amino acids 380-603) of HIF-1a to the a chain of the mcCAR, thereby conferring oxygen-dependent stability on the fusion receptor. In HIF-CAR2, the N-terminal domain containing the key proline residue $\mathrm{P} 4 \mathrm{O} 2$ was added (amino acids 344-417), while in HIF-CAR3, the C-terminal domain containing the key proline residue P564 (amino acids 530-652) was added. As indicated above, these residues have previously been shown to interact with the VHL tumour-suppressor protein and to be hydroxylated in conditions of physiological normoxia, ultimately leading to protein degradation. ${ }^{42-44}$

To demonstrate that oxygen could be used to turn on the switch, $T$ cells were electroporated with mRNA encoding for the CAR-ODD for comparison with a control CAR that lacked an ODD. After incubation for 20 hours under hypoxic conditions, cell-surface expression of HIF-CAR1 and HIF-CAR2 were enhanced, while the HIF-CAR3 (for unclear reasons) and the control CAR responded minimally. To demonstrate that the switch was reversible, $T$ cells were transferred from hypoxia to normoxia for 6 hours. Cell-surface expression of HIF-CAR1 and HIF-CAR2 were both successfully negated, whereas no change in expression was observed for the control CAR.

To further evaluate the dynamics of the system and the rate of CAR decay upon removal from hypoxia, $T$ cells were electroporated and placed in normoxia for 6 hours to recover. Next, hypoxia was applied for 16 hours and cellsurface CAR expression was monitored over 6 hours of reoxygenation thereafter. Cell-surface CAR expression decreased by $80 \%$ with a lapse of 2 hours for both HIF-CAR1 and HIF-CAR2 and this remained stable for the next 4 hours. In concordance with previous experiments, control CAR and HIF-CAR3 did not respond upon reoxygenation.

To assess cytolytic activity, control or HIF-CAR1 $T$ cells were co-cultured at different effectorto-target ratios for 16 hours with Daudi cells (a human Burkitt lymphoma B-cell line), either in normoxic or hypoxic conditions. Tumour cell killing was observed in both hypoxic and normoxic conditions at effector-to-target ratios of 5:1 and 10:1, respectively. While enhanced killing was seen in hypoxic conditions, leakiness of the system was suggested by the detection of cytotoxic activity under conditions of normoxia. ${ }^{40}$ 


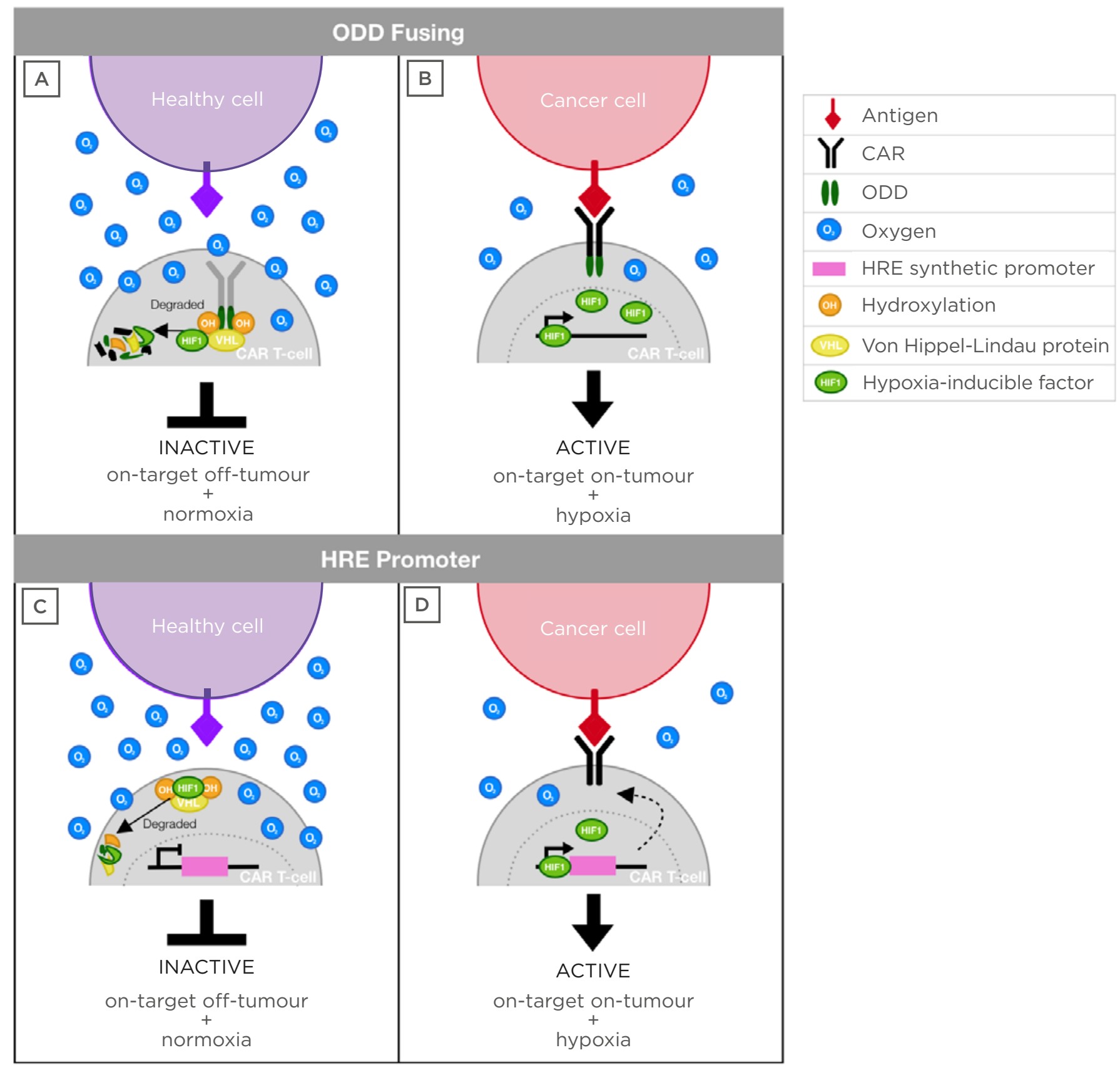

Figure 1: Schematic to illustrate hypoxia-sensing CAR-T cell approaches.

A,B) ODD fusion approach. Upon infusion, CAR-T cells may engage healthy (A) or cancer (B) cells that express cognate target. When the CAR is exposed to normoxia, the ODD fused to the CAR become hydroxylated and recognised by the $\mathrm{VHL}$, which ubiquitinates the CAR for proteasomal degradation. In hypoxia (B), prolyl hydroxylase does not hydroxylate the CAR, which prevents degradation and allows target-dependent CAR activation.

C,D) HRE-promoter approach. Upon infusion, CAR-T cells situated in healthy tissue (C) are exposed to normoxia and thus CAR transcription is not triggered, protecting healthy cells that express the antigen and avoiding on-target, offtumour toxicity. By contrast, HIF-1 complex accumulation occurs selectively in conditions of hypoxia (D), allowing the binding of this complex to HRE in the synthetic vector promoter region. As a result, CAR expression occurs, which enables the activation of these $T$ cells upon antigen binding.

CAR: chimeric antigen receptor; HIF-1: hypoxia-inducible factor 1; HRE: hypoxia-responsive elements; ODD: oxygendependent degradation domain; VHL: von Hippel-Lindau protein. 


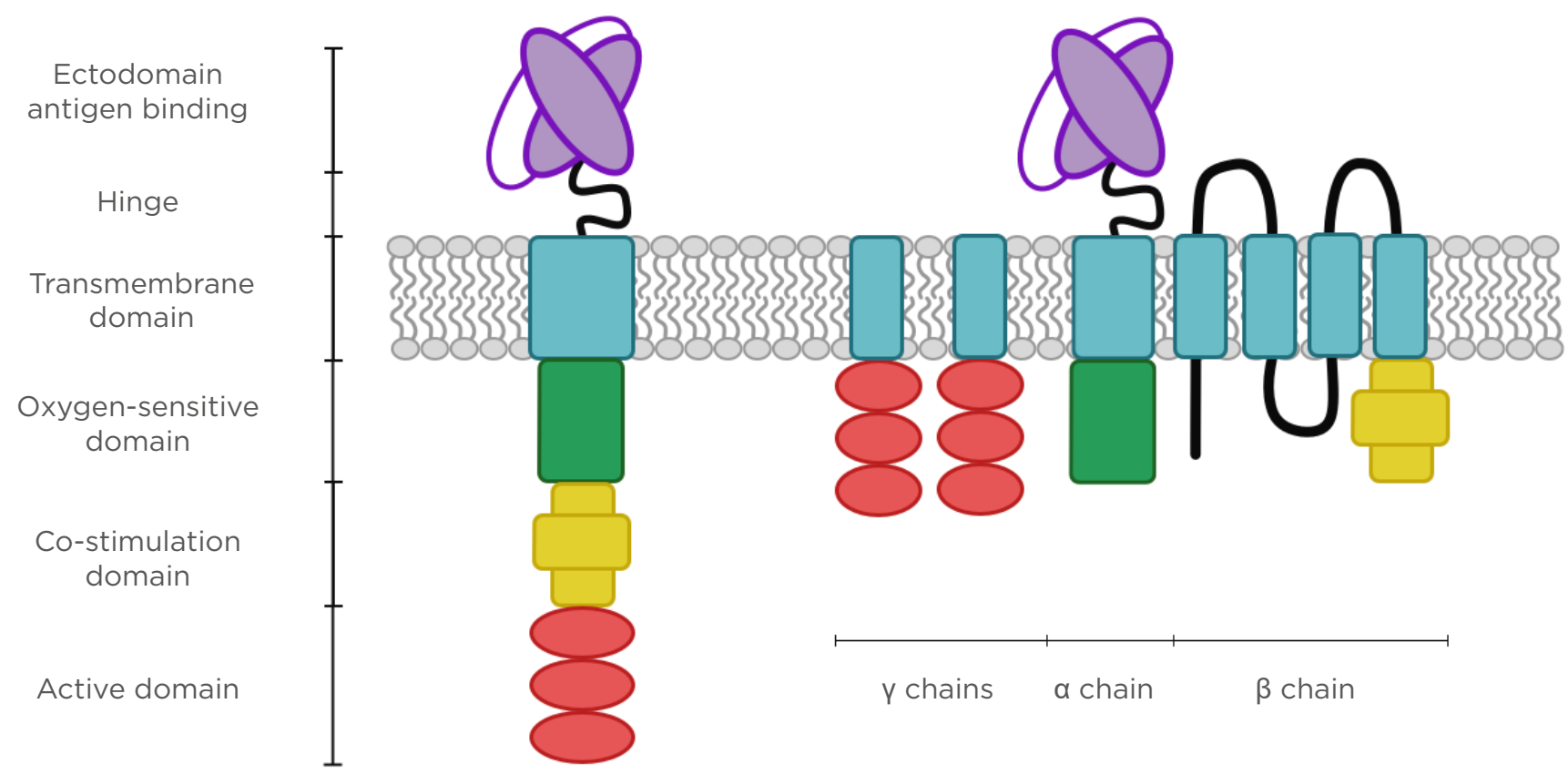

A Single-chain CAR

B Multichain CAR

Figure 2: Multi-input CAR designs.

A) Single-chain CAR. The targeting, activation, co-stimulation, and ODD domains are fused in a unique chain.

B) Multichain CAR. The different domains are distributed in three different chains ( $\alpha, \beta$, and $\gamma$ ).

CAR: chimeric antigen receptor; ODD: oxygen-dependent degradation domain.

The strategies described thus far have taken advantage of the HIF master orchestrators of oxygen responsiveness, through the fusion of an ODD to the CAR endodomain (Figure 1AB). An alternative oxygen-sensing CAR system was recently presented by Sarén et al. ${ }^{45}$ in an abstract. They fused a cassette of hypoxia-responsive elements to a minimal cytomegalovirus promoter and evaluated the ability of this putative hypoxia-responsive promoter to drive expression of a CD19-specific CAR (Figure 1C-D). Following chemical induction of hypoxia, they showed that expression of the CAR was enhanced, whereas the expression of a control CAR under the transcriptional control of a constitutive EFla promoter was unaltered. Additionally, the group reported that the oxygensensing CAR displayed enhanced activation and cytotoxic activity upon antigen recognition in conditions of hypoxia, when compared to normoxia. ${ }^{45}$

\section{CONCLUSIONS AND PERSPECTIVE}

The aforementioned studies provide proof of concept as to how hypoxia may be exploited to enhance precision targeting of CAR-T cell immunotherapy.

A further theoretical advantage relates to the fact that restricted CAR expression at sites of hypoxia might be expected to mitigate against the deleterious effects of tonic signalling, which is a particular problem with some singlechain variable fragment-targeted CAR. ${ }^{46}$ Moreover, T cells that escape from the tumour microenvironment should be rapidly disarmed owing to CAR ubiquitination under oxygenreplete conditions. By this means, off-tumour ontarget toxicity should be minimised. It should be acknowledged, however, that ODD-dependent CAR degradation can take between minutes and hours ${ }^{47}$ meaning that some such toxicity might nonetheless occur. 
In the further advancement of these technologies, a number of points warrant consideration. While physiological oxygen levels vary across many normal tissues, they are lower than atmospheric oxygen levels. This highlights the need for careful testing of CAR expression and function across a range of oxygen tensions. Another potential concern is that the activity of hypoxia-sensing CAR could be suboptimal in tumours with heterogeneous oxygen levels, owing to excessive CAR degradation in physiological normoxic areas. ${ }^{47}$ Finally, leakiness of some systems could prove problematic in the clinical application of this technology. While it has been argued that low-level CAR expression under oxygen-replete conditions could help initiate CAR-T cell activation, ${ }^{40}$ concerns remain that on-target, off-tumour toxicity could occur as a result. Additionally, inflammation triggered by low-level CAR activation might be expected to cause further local hypoxia, creating a vicious circle in which further CAR upregulation, tissue damage, and inflammation could ensue. By contrast, systems that rely exclusively on HIFtriggered CAR transcription may be undermined in part by the fact that TCR activation can also trigger HIF stabilisation via mTOR signalling. ${ }^{48}$

In summary, highly stringent restriction of CAR expression to the tumour microenvironment constitutes a desirable AND gate by which to restrict CAR function at the site of disease (e.g., where target and hypoxia colocalise). However, with currently available systems, this remains an unmet goal that requires further technological refinement. The successful development of such a technology would broaden extensively the range of targets and cell dose levels that could be safely employed in efforts to develop effective CAR-T cell immunotherapies for solid tumours.

\section{References}

1. Kosti $\mathrm{P}$ et al. Perspectives on chimeric antigen receptor $\mathrm{T}$-cell immunotherapy for solid tumors. Front Immunol. 2018;9:1104.

2. Magalhaes I et al. The metabolic profile of tumor and virally infected cells shapes their microenvironment counteracting $T$ cell immunity. Front Immunol. 2019;10:2309.

3. Bonifant $\mathrm{CL}$ et al. Toxicity and management in CAR T-cell therapy. Mol Ther Oncolytics. 2016;3:16011.

4. Yazdanifar $M$ et al. Emerging immunotherapeutics in adenocarcinomas: A focus on CAR-T cells. Curr Trends Immunol. 2016;17:95-115.

5. Piscopo NJ et al. Bioengineering solutions for manufacturing challenges in CAR T cells. Biotechnol J. 2018;13(2):10.1002/biot.201700095

6. Papa $\mathrm{S}$ et al. Clinical evaluation of ErbB-targeted CAR T-Cells, following intracavity delivery in patients with ErbB-expressing solid tumors. Methods Mol Biol. 2015;1317:365-82.

7. Gross G, Eshhar Z. Therapeutic potential of $T$ cell chimeric antigen receptors (CARs) in cancer treatment: counteracting off-tumor toxicities for safe CAR T Cell Therapy. Annu Rev Pharmacol Toxicol. 2016;56:59-83.

8. Tchou J et al. Safety and efficacy of intratumoral injections of chimeric antigen receptor (CAR) $T$ cells in metastatic breast cancer. Cancer Immunol Res. 2017;5(12):1152-61.
9. Papa $\mathrm{S}$ et al. A phase I trial of T4 CAR T-cell immunotherapy in head and neck squamous cancer (HNSCC). J Clin Oncol. 2018;36(Suppl 15):3046.

10. Di Stasi A et al. Inducible apoptosis as a safety switch for adoptive cell therapy. N Engl J Med. 2011;365(18):1673-83.

11. Fedorov VD et al. PD-1- and CTLA4-based inhibitory chimeric antigen receptors (iCARs) divert off-target immunotherapy responses. Sci Transl Med. 2013;5(215):215ra172.

12. Wilkie $S$ et al. Dual targeting of ErbB2 and MUC1 in breast cancer using chimeric antigen receptors engineered to provide complementary signaling. J Clin Immunol. 2012;32(5):1059-70.

13. Roybal KT et al. Precision tumor recognition by $T$ cells with combinatorial antigen-sensing circuits. Cell. 2016;164(4):770-9.

14. Juillerat $A$ et al. Design of chimeric antigen receptors with integrated controllable transient functions. Sci Rep. 2016;6: 18950

15. Rodgers DT et al. Switch-mediated activation and retargeting of CAR-T cells for B-cell malignancies. Proc Nat Acad Sci U S A. 2016;113(4):E459-68.

16. Raj D et al. Switchable CAR-T cells mediate remission in metastatic pancreatic ductal adenocarcinoma. Gut. 2019;68(6):1052-64.

17. Giordano-Attianese $\mathrm{G}$ et al. A computationally designed chimeric antigen receptor provides a small-molecule safety switch for T-cell therapy. Nat Biotechnol. 2020;38(4):426-32.

18. Schurich $A$ et al. Metabolic regulation of CAR T cell function by the hypoxic microenvironment in solid tumors. Immunotherapy. 2019;11(4):335-45.

19. Nordsmark M et al. Prognostic value of tumor oxygenation in 397 head and neck tumors after primary radiation therapy. An international multi-center study. Radiother Oncol. 2005;77(1):18-24.

20. Rademakers SE et al. Molecular aspects of tumour hypoxia. Mol Oncol. 2008;2(1):41-53.

21. Cosse J-P, Michiels C. Tumour hypoxia affects the responsiveness of cancer cells to chemotherapy and promotes cancer progression. Anticancer Agents Med Chem. 2008;8(7):790-7.

22. Brown JM, Wilson WR. Exploiting tumour hypoxia in cancer treatment. Nat Rev Cancer. 2004;4(6):437-47.

23. Semenza GL et al. Hypoxia-inducible nuclear factors bind to an enhancer element located 3 ' to the human erythropoietin gene. Proc Natl Acad Sci U S A. 1991;88(13):5680-4.

24. Maxwell PH et al. The tumour suppressor protein VHL targets hypoxia-inducible factors for oxygendependent proteolysis. Nature. 1999;399(6733):271-5.

25. Ivan M et al. HIFa targeted for VHLmediated destruction by proline 
hydroxylation: implications for $\mathrm{O} 2$ sensing. Science. 2001;292(5516):464-8.

26. Semenza GL. Life with oxygen Science. 2007;318(5847):62-4

27. Semenza GL. Surviving ischemia: adaptive responses mediated by hypoxia-inducible factor 1 . J Clin Invest. 2000;106(7):809-12.

28. Wenger $\mathrm{RH}$ et al. Integration of oxygen signaling at the consensus HRE. Sci STKE. 2005:2005(306):re12.

29. Wilson WR, Hay MP. Targeting hypoxia in cancer therapy. Nat Rev Cancer. 2011:11(6):393-410.

30. Brown JM. SR 4233 (Tirapazamine) a new anticancer drug exploiting hypoxia in solid tumours. Br J Cancer. 1993;67(6):1163-70.

31. Jeong $W$ et al. Pilot trial of EZN-2968, an antisense oligonucleotide inhibitor of hypoxia-inducible factor-1 alpha (HIF-1 $\alpha$ ), in patients with refractory solid tumors. Cancer Chemother Pharmacol. 2014;73(2):343-8.

32. Carraro F et al. p66Shc is involved in promoting HIF-1a accumulation and cell death in hypoxic T cells. J Cell Physiol. 2007:211(2):439-47.

33. Zenewicz LA. Oxygen levels and immunological studies. Front Immunol. 2017:8:324.

34. Xu Y et al. Glycolysis determines dichotomous regulation of $\mathrm{T}$ cell subsets in hypoxia. J Clin Invest. 2016;126(7):2678-88.

35. Gropper $Y$ et al. Culturing CTLs under hypoxic conditions enhances their cytolysis and improves their anti-tumor function. Cell Rep. 2017;20(11):2547-55

36. Berahovich $\mathrm{R}$ et al. Hypoxia selectively impairs CAR-T cells in vitro. Cancers (Basel). 2019;11(5):602.

37. Ang SO et al. Conditional T-cell activation for tumor under hypoxia. Blood. 2008;112(11):3906

38. Chan DA et al. Coordinate regulation of the oxygen-dependent degradation domains of hypoxiainducible factor 1 Alpha. Mol Cell Biol. 2005;25(15):6415-26.

39. Ang SO et al. Conditional Activation of T Cells to Specifically Target c-Met under Hypoxia. Abstract 62. American Society of Gene Therapy $12^{\text {th }}$ Annual Meeting, 27-30 May, 2009.

40. Juillerat $A$ et al. An oxygen sensitive self-decision making engineered CAR T-cell. Sci Rep. 2017:7(39833):1-8.

41. Kinet JP. The high-affinity IgE Receptor (Fc》RI): from physiology to pathology. Annu Rev Immunol. 1999;17:931-72.

42. Masson $\mathrm{N}$ et al. Independent function of two destruction domains in hypoxia-inducible factor- $\alpha$ chains activated by prolyl hydroxylation. EMBO J. 2001:20(18):5197-206.

43. Epstein ACR et al. C. elegans EGL-9 and mammalian homologs define a family of dioxygenases that regulate HIF by prolyl hydroxylation. Cell. 2001:107(1):43-54.

44. Paltoglou S, Roberts BJ. HIF-1a and EPAS ubiquitination mediated by the VHL tumour suppressor involves flexibility in the ubiquitination mechanism, similar to other RING E3 ligases. Oncogene. 2007;26(4):604-9

45. Sarén TA et al. Hypoxia-responsive CAR T-cells. Abstract A041. $4^{\text {th }}$ CRI-CIMT-EATI-AACR International Cancer Immunotherapy Conference, 30 September-3 October, 2018

46. Ajina A, Maher J. Strategies to address chimeric antigen receptor tonic signaling. Mol Cancer Ther. 2018;17(9):1795-815

47. Caruso HG et al. Steering CAR $T$ cells to distinguish friend from foe. Oncoimmunology. 2019;8(10):e1271857.

48. Liu $C$ et al. mTOR and metabolic regulation of conventional and regulatory T cells. J Leukoc Biol. 2015:97(5):837-47. 